R1823

\title{
Circulating Irisin Level as an Early Biomarker of Decline of Metabolic Health
}

Massimiliano Marco Corsi Romanelli ${ }^{1,2}$, Elena Vianello ${ }^{1}$, Clementina Sitzia ${ }^{1}$, Stefano Benedini ${ }^{1}$, Silvia Gorini ${ }^{3}$, Benedetta Rampoldi ${ }^{2}$, Roberta Rigolini ${ }^{2}$, Lorenza Tacchini ${ }^{1}$, Federico Ambrogi ${ }^{1}$, Elena Dozio ${ }^{1}$

${ }^{1}$ Università degli Studi di Milano, ${ }^{2}$ IRCCS Policlinico San Donato, ${ }^{3}$ Instrumentation Laboratory - A Werfen Company

A decline in metabolic health may take place before observing an alteration in the levels of the traditional metabolic markers. New indicators of metabolic derangement are therefore compelling. Irisin is a myokine which exerts important metabolic functions: it increases energy expenditure, improves insulin resistance, reduces glucose level and advanced glycation end products (AGE)-induced inflammation. The optimal level for irisin in human and its potential role as a metabolic biomarker have not been fully established yet.

To this aim, we quantified plasma irisin level in 106 healthy subjects (age $43 \pm 11.72$ years, 58 males/ 48 females) and we performed a cluster analysis to evaluate the association between irisin and subject characteristics [age, total cholesterol, LDL, HDL, tryglicerides, NEFA (non-esterified free fatty acids), glucose, HbAlc (glycated hemoglobin), insulin, GA (glycated albumin), uric acid, creatinine, irisin, sRAGE (total soluble receptor for AGE), esRAGE (endogenously secreted receptor for AGE), cRAGE (membrane-cleaved receptor for AGE) and cRAGE/esRAGE ratio]. ELISA tests were used for the quantification of parameters different from routine biochemical markers. Glycated albumin (GA) was meausured by the enzymatic method Lucica ${ }^{\circledR G A}-\mathrm{L}$. The study was conducted in accordance with the Declaration of Helsinki.

Irisin median level was $7.89 \mathrm{ng} / \mathrm{ml}$. Although the mean levels of the traditional metabolic blood markers were within the ranges of normality, subjects in cluster 1 displayed a metabolic profile shifted toward the highest reference values. In fact, we observed higher concentrations of total cholesterol, LDL, triglycerides, NEFA, glucose, HbAlc, insulin, uric acid and creatinine. The reduced levels of the anti-AGE molecules sRAGE and esRAGE, the increased levels of cRAGE/esRAGE (a surrogate marker of inflammation) and GA (an AGE product), and a greater percentage of subjects with visceral obesity in cluster 1 confirmed an increased metabolic stress and a pro-inflammatory profile in this group. Irisin concentration was higher in this group too.

The traditional metabolic blood tests seem to be not enough to early identify a metabolic decline. The increase of irisin level may reflect a metabolic derangement at the beginning of its development. The role of irisin as an early biomarker of decline in metabolic health seems an interest topic to be further explored.

\section{Support or Funding Information}

The study was supported by funding from Fondazione E. A. Fiera Internazionale di Milano to Università degli Studi di Milano and Ricerca Corrente funding from Italian Ministry of Health to IRCCS Policlinico San Donato. 